\title{
Carrying metazoan phylogenetics forward in the 21st century
}

\author{
Ronald A. Jenner \\ Institute for Biodiversity and Ecosystem Dynamics, University of Amsterdam, P.O. Box 94766, 1090 GT \\ Amsterdam,The Netherlands.E-mail: jenner@science.uva.nl
}

Review of and commentary on: Animal Evolution. Interrelationships of the Living Phyla, by Claus Nielsen. Oxford University Press, 2001, x + 563 pp., ISBN 0-19-850682-1 (Pbk).

My initial browsing of the new edition of Claus Nielsen's Animal Evolution could scarcely have produced a bigger surprise. Opening the book from the back I first read the last line of the last chapter on molecular phylogeny: "The evolutionary scenario pictured in fig. 57.2 [a molecular cladogram based on $18 \mathrm{~S}$ rDNA sequences] is fully compatible with the phylogeny proposed on morphological evidence in this book..." (p. 519). Imagine the surprise! Anyone even marginally familiar with recent developments in higher-level animal phylogenetics will be aware of some major conflicting phylogenetic signals lurking below the surface of morphological and molecular data sets. Certainly, many may agree with Balter's (1997) observation that "morphologists learn to live with molecular upstarts," but in view of Nielsen's less than enthusiastic embrace of molecular phylogenetic evidence in previous publications (Nielsen, $1997 \mathrm{a}, \mathrm{b})$ he appears particularly unlikely to serve as a candidate molecular convert. However, a glance at Nielsen's molecular "tree" offers immediate clarification. It resembles a three-layered terrace overgrown with a phylogenetic grass reminiscent of the massively polyphyletic scheme advocated a decade ago by Willmer (1990), which enjoyed some notoriety as the then most recent pictorial incarnation of phylogenetic ignorance. Nielsen's rendition of $18 \mathrm{~S}$ rDNA sequence data merely yields three phylogenetic plateaus representing the ascending organizational levels of Metazoa, Bilateria, and Deuterostomia forming the substrates of three successive Precambrian radiations. Unsurprisingly, the complete lack of resolution in the molecular phylogeny-guarantees compatibility with any morphological tree. Needless to say, others have reached rather different conclusions about the degree of phylogenetic resolution that $18 \mathrm{~S}$ data can offer. In fact, where most phylogeneticists (including myself) are prepared to defend more resolution for inter-phylum relationships, Nielsen's molecular cladogram offers resolution where $18 \mathrm{~S}$ data do not provide it, namely for the monophyly of several of the individual phyla. Nielsen's fig. 57.1 nicely illustrates this shortcoming of $18 \mathrm{~S}$ data to support the monophyly of even the morphologically bestdefined phyla such as Mollusca. Nielsen very briefly touches upon some other issues with molecular phylogenetics that have attracted widespread attention in the recent literature, such as the difficulty of estimating divergence times, and the conflict or congruence between phylogenetic estimates based on different molecules. A short section on evolutionary developmental biology is also provided, but readers who are seriously interested in molecular phylogenetics are best advised to consult the extensive primary literature as Nielsen himself candidly recommends.

The chapter on molecular phylogeny is just one of six new chapters in this new edition of the 1995 original. The book has retained the overall attractive layout of the first edition. For readers not acquainted with Nielsen's book, it is a compre- 
hensive phylogenetic analysis of the living animal phyla, based upon a detailed study of their comparative morphology. Every phylum is considered in a separate chapter that offers lucid descriptions of its morphology and embryology emphasizing phylogenetically informative features. The monophyly of each phylum is justified, and ancestral character states are assumed or reconstructed through a phylogenetic analysis of intra-phylum relationships. These plesiomorphic character states form the basis for the phylogenetic analyses presented in the book. Throughout the book Nielsen carries out manual phylogenetic analyses of restricted sets of phyla that make up the larger clades in his phylogeny, while chapter 56 reports the results of a computer-assisted cladistic analysis of all phyla analysed simultaneously. Adequate illustrations support the text throughout the book, but the reader is well-advised to have a textbook on invertebrate zoology ready at hand to provide extra pictorial support for the dense morphological narrative. Apart from the chapters on molecular phylogeny (57) and numerical cladistic analyses (56) three other new chapters $(9,30,32)$ accompany the shifting phylogenetic positions of ctenophores and gnathostomulids, the latter now receiving a separate chapter. Four chapters from the first edition were "removed." The two chapters in the first edition $(2,56)$ discussing the trochaea theory are now partly replaced by the new chapter 3 dealing with early animal radiation. The old chapter on Acanthocephala is now merged with that on the Rotifera. The original 1995 chapter on "Five enigmatic taxa" has changed its membership somewhat: Xenoturbella is now placed within Mollusca, while the newly described cycliophoran Symbion pandora is treated as incertae sedis. Finally, Nielsen has globally updated the information in the book, removed errors, streamlined the text in various places, and added several new illustrations. New morphological information and novel character interpretations have changed the topology of the phylogeny somewhat since the first edition. In conclusion, Nielsen has written a wonderful book that despite the treatment of a great amount of detail is characterized by a high degree of factual accuracy, and that amply justifies the frequent citation of the first edition.

A burning question remains: has Nielsen pro- duced the definitive morphological tree of the Metazoa? Clearly, his phylogeny is the result of a penetrating analysis of a vast quantity of information by a zoologist in total command of the comparative morphology of extant metazoans. However, there is no shortage of alternative morphological phylogenies. Consider the four computerassisted cladistic analyses (Giribet et al,, 2000; Sørensen et al., 2000; Peterson \& Eernisse, 2001; Zrzavý et al., 2001) that were published since last year! Although parts of these trees agree, many differences in topology remain.

Unfortunately, Nielsen has chosen not to discuss the relative merits of published numerical cladistic studies. Instead, he offers several remarks that echo more than a whisper of despair over the challenging task that is still before us: 1) explain what causes the differences in topology between different analyses, and 2) identify the best supported tree(s). On p. 499 we read that in contrast to phylogenetic analyses of lower-level taxa, such as "species, and perhaps also genera" where the data matrix "can possibly be made objective," grim prospects await those who wish to perform objective phylogenetic analyses of higher-level taxa because "the choice and definition of taxa and choice and coding of characters become a complete quagmire," and "character codings will to some degree be personal, i.e. subjective." Are we to believe that an objective evaluation of alternative cladistic hypotheses is doomed to failure and not worthy of our time? I hope not. The outcome of any phylogenetic analysis is interpreter-dependent or subjective, but it is essential to recognize that this is a characteristic shared amongst all historical sciences ( $O$ 'Hara, 1992), and it does not in principle prevent an objective analysis of historical events. The key hallmarks of objective science are explicitness and the possibility of testing. Selection of taxa and characters, and decisions of primary homology may differ between analyses, sometimes even extensively, but these variables can be objectively compared between studies. A comparison of these variables may then form the basis for an objective judgement on the relative merit of different analyses. Even if no single hypothesis can be nominated as the superior summary of available evidence, we can nevertheless identify those decisions that are 
most decisive in determining conflicting topologies, and design future research accordingly. For example, different analyses may have proposed either taxon $\mathrm{B}$ or $\mathrm{C}$ as a sister group of taxon $\mathrm{A}$. When a study aimed at testing these alternatives includes only taxa $A$ and $B$, we must conclude that restrictive taxon selection has not allowed a true test of available alternatives. Thus the objectivity of phylogenetic analyses resides in the explicitness of the data matrix.

As a concrete example, consider the position of platyhelminths in Nielsen's tree (fig. 56.1) while focusing on just one analysis parameter: character selection. Platyhelminthes and Nemertea are sister taxa forming the clade Parenchymia. Nielsen's cladistic analysis found one unambiguous apomorphy: 'larva with strongly reduced hyposphere' (the part of the larva posterior to the band of long cilia is reduced in size). When we examine other recently published cladistic analyses, we find that no less than 20 different characters have been proposed as diagnostic for conflicting sister group relationships of platyhelminths. None of these characters are included in Nielsen's new analysis, and conversely none of the other studies included Nielsen's parenchymian apomorphy! This clearly shows that differences in character selection contribute substantially to the existence of conflicting phylogenetic placements of platyhelminths. For an effective character congruence test of the alternatives, future studies must carefully select all pertinent characters so that no potential outcomes are precluded $a$ priori. Of course, before the most likely sister group(s) to platyhelminths can be identified with confidence, the other variables that differ between the studies will have to be scrutinized as well.

Interestingly, Nielsen's discussion of platyhelminths and nemerteans provides a hint that is helpful in further evaluating the confidence we may have in the parenchymian apomorphy. Nielsen (p. 508) writes that the "numerical analyses show a reasonable accordance with the 'manual' analysis performed throughout this book, indicating that obvious logical inconsistencies between the interpretation of characters and the construction of phylogeny are not present." However, the character "larva with strongly reduced hyposphere" is based on similarities between the polyclad Götte's and the nemer- tean pilidium larvae, which assumes that these larval forms are ancestral for the respective monophyletic phyla. In a set of apparently contradictory statements, Nielsen then concludes not only that the pilidium larva "is close to the original type" (p. 264), but also that "the life cycle involving a pilidium larva is definitely not ancestral" (p. 288, my italics). You cannot have it both ways. Either the pilidium larva is a unique novelty that evolved within a monophyletic Nemertea, thereby logically precluding any phylogenetic relevance it might have for linking nemerteans to other phyla, or the pilidium is truly representative of the original nemertean larva permitting a search for phylogenetically informative similarities with other larval forms.

Another example of internal inconsistency in Nielsen's book is his conclusion that rotifers must be interpreted as "neotenic descendants of the protostomian ancestor, gastroneuron" (p. 305). This statement stands in direct conflict with the phylogenies defended in the book. The gastroneuron and the most recent common ancestor of Rotifera are separated by either three or five different ancestral nodes depending upon whether one chooses to consult either Nielsen's manual, or computer-generated phylogeny. This discrepancy demands either a revision of Nielsen's phylogenies, or a reconsideration of an attractive but highly speculative theory about rotifer body plan evolution. The latter option seems preferable at this time because no phylogenetic analysis published to date (based upon morphology, molecules, or combined evidence) indicates a position for rotifers immediately at the base of the protostomes.

Morphological approaches to metazoan phylogeny are extremely challenging. We attempt to reconstruct diversification events that took place more than half a billion years ago on the basis of scattered information that is imperfectly preserved in the complex structure, development and molecular architecture of living animals, while information from fossils is habitually excluded from cladistic analyses of the Metazoa. Given such circumstances, multiple alternative hypotheses are a logical expectation. Importantly, the congruence that we do find between different morphological and molecular phylogenetic studies attests to the tractability of this difficult task. However, at this time it is prac- 
tically impossible to make a reasoned choice between phylogenies, and it is a dangerous rule-ofthumb to just pick the newest or biggest analysis (Jenner, 2001).

Although the many "subjective" decisions that feed into different cladistic studies may appear to Nielsen as a "complete quagmire," they do not form a Gordian knot. Detailed comparative study of alternative phylogenetic hypotheses will have to take center-stage as we enter the 21 st century. This will require at least some change in contemporary research strategies. Recent cladistic studies of the Metazoa have been typically concerned with in depth analyses of single morphological data matrices (either alone or in combination with molecular sequence data) with only minimal (if any) attention paid to exploring the robustness and reliability of their results relative to those of other studies. In order to achieve further progress in this field of phylogenetic research we have to shift our focus towards the detailed comparative study of the accumulated phylogenies. Only this will allow us to conclude which trees we can refute or provisionally accept pending further study.

Nielsen's masterful synthesis will be an indispensable tool in such an endeavor, and the book is required reading for anybody interested in the fascinating problems of higher-level animal relationships and body plan evolution.

\section{References}

Ax P. 2000. Multicellular animals. The phylogenetic system of the Metazoa. Springer-Verlag, Berlin.

Balter M. 1997. Morphologists learn to live with molecular upstarts. Science 276: 1032-1034.

Giribet G, Distel DL, Polz M, Sterrer W, Wheeler WC. 2000. Triploblastic relationships with emphasis on the acoelomates and the position of Gnathostomulida, Cycliophora, Plathelminthes, and Chaetognatha: a combined approach of 18S rDNA sequences and morphology. Syst. Biol. 49: 539-562.

Jenner RA. 2001. Bilaterian phylogeny and uncritical recycling of morphological data sets. Syst. Biol. 50: 730-742.

Nielsen C. 1997a. Animal classification - then and now. Bull. Soc. zool. Fr. 122: 243-253.

Nielsen C. 1997b. The phylogenetic position of the Arthropoda. In: Fortey RA, Thomas RH, eds. Arthropod relationships. The Systematics Association Special Volume Series 55. London, Chapman \& Hall, 11-22.

O'Hara RJ. 1992. Telling the tree: narrative representation and the study of evolutionary history. Biol. Phil. 7: 135160.

Peterson KJ, Eernisse DJ. 2001. Animal phylogeny and the ancestry of bilaterians: inferences from morphology and 18S rDNA gene sequences. Evo. Devel. 3: 170-205.

Sørensen MV, Funch P, Willerslev E, Hansen AJ, Olesen J. 2000. On the phylogeny of the Metazoa in light of the Cycliophora and Micrognathozoa. Zool. Anz. 239: 297318.

Willmer P. 1990. Invertebrate relationships. Patterns in animal evolution. Cambridge, Cambrìdge University Press.

Zrzavý J, Hypša V, Tietz DF. 2001. Myzostomida are not annelids. Molecular and morphological support for a clade of animals with anterior sperm flagella. Cladistics 17: 170-198.

Received: 2 October 2001 